\title{
ANÁLISIS Y PROPUESTA DE UN MODELO DE VIRTUALIZACIÓN DE LA UNMSM. INNOVACIÓN, VIRTUALIZACIÓN Y FLEXIBILIZACIÓN CURRICULAR
}

\author{
Aquiles Bedriñana Ascarza * \\ E-mail:aquiles_unmsm@hotmail.com \\ Daniel Rincón Prada *** \\ E-mail: ... \\ William Aguayo Mucha ***** \\ E-mail:waguayom_af@unmsm.edu.pe
}

\begin{abstract}
RESUMEN
Como estrategia para el proceso de modernización e innovación educativa de la Universidad Nacional Mayor de San Marcos (UNMSM), la decana de América, es imperativo emprender el proceso de virtualización de la Universidad, con perspectivas a su universalización para un mundo mejor.

El objetivo general de este estudio es el de diseñar un modelo de virtualización para innovar los procesos de formación presencial y de Experiencias de Educación a Distancia (EaD), así como las actividades de investigación y extensión universitaria en la UNMSM. Uno de los objetivos específicos del proyecto es el de evaluar la factibilidad técnica, económica y pedagógica para el desarrollo de un sistema de enseñanza electrónico (e-learning) en la UNMSM.

Primeramente se analiza el estado actual, alternativas y perspectivas de la educación virtual en el Perú, las ventajas y desventajas del e-learning como soporte de la educación presencial y a distancia. Además, se realiza un estudio sobre las tecnologías existentes en el mercado para el proceso de selección de plataformas Learning Management System (LMS) y de Gestión de Contenidos (Learning Content Management System - LCMS), señalando los requisitos técnicos, pedagógicos y administrativos que debe reunir una plataforma tecnológica.
\end{abstract}

Para la virtualización progresiva de la UNMSM se presenta una propuesta de un modelo pedagógico y organizacional adecuado a la realidad de nuestra alma máter.

Palabras clave: Virtualización, e-learning, EaD, educación virtual y Modelo Pedagógico, Organizacional y Tecnológico.

* Doctor en Educación. Magíster en Ingeniería de Sistemas y MBA. Profesor Principal e Investigador Permanente de la Unidad de Investigación. Past-Decano de la Facultad de Ciencias Administrativas (UNMSM).

粎 Licenciado en Administración. 


\begin{abstract}
As strategy for the process of Educational Modernization and Innovation of the Universidad Nacional Mayor de San Marcos (UNMSM), the dean of America, is imperative to undertake the process of virtualización of the University, with perspectives its universalization for a better world.

The general objective of this study is the design of a model of virtualización to innovate the processes of eyewitness formation and of Experiences of Distance Education, as well as the activities of investigation and university extension in the UNMSM. One of the specific objectives of the project is the evaluation of pedagogical, economic, and technical factibility for the development of an electronic educational system (e-learning) in the UNMSM.

First, we examine the current state, alternatives and perspectives of the virtual education in Peru, the advantages and disadvantages of e-learning as support of eyewitness and distance education. Besides, a study on the existing technologies in the market for the platforms selection process is carried out Learning Management System (LMS) and Contents Management (Learning Content Management System - LCMS), indicating the administrative, pedagogical, and technical requirements that should needs a technological platform.

For the Progressive Virtualización of UNMSM a proposal of a pedagogical and organizational model is presented adequate to the reality of our alma mater.
\end{abstract}

Key words: Virtualization, e-learning, EaD, virtual education and Pedagogical Model, Organizacional and Technological.

\section{INTRODUCCIÓN}

En los últimos años se ha acelerado el fenómeno de virtualización de la docencia universitaria, como un reflejo de la presencia extensiva e intensiva de las Nuevas Tecnologías de Información y Comunicación (NTIC) que vienen generando cambios paradigmáticos en la educación superior. Las universidades de los países en desarrollo enfrentan el desafío de servir a una población cada vez mayor de estudiantes, más diversificada social y culturalmente, en un nuevo ambiente cada vez más dinámico.

La virtualización (parcial o total) de estas organizaciones puede ser un factor transformador de sus estructuras y funciones, un instrumento para mejorar su cobertura, calidad, pertinencia y equidad de acceso, que permita construir una nueva identidad en la Sociedad del Conocimiento.

Como estrategia para el proceso de modernización e innovación educativa de la Universidad Nacional Mayor de San Marcos se puede emprender su proceso de virtualización para que se transforme en una universidad abierta que permita su universalización para un mundo mejor.

La virtualización de universidades y programas tradicionales a distancia en Iberoamérica se viene dando progresivamente. Desde 1972 se experimenta el crecimiento de universidades $y$, especialmente, de programas de educación superior a distancia, como se observa en la región Iberoamericana. En efecto, pueden señalarse entre otras, las siguientes universidades: Universidad Nacional de Educación a Distancia (UNED) y la Universitat Oberta de Cataluyna (UOC), ambas en España; la Universidad Estatal a Distancia (UNED) de Costa Rica y la Universidad Abierta de Loja, en Ecuador.

Pero además de estas instituciones, dedicadas exclusivamente a la modalidad a distancia, han surgido centenares de otros programas a distancia, ubicados generalmente dentro de tradicionales estructuras de universidades presenciales. De estos numerosos programas tenemos al Sistema de Universidad Abierta (SUA) en la Universidad Autónoma de México, pionero en su género en América Latina; y el de la Facultad de Educación a Distancia, que forma parte de la Universidad de La Habana, Cuba.

En el caso peruano, los diagnósticos sobre las experiencias de educación a distancia $(\mathrm{EaD})$ demuestran que ella es cada vez mas aceptada cuando incorporan las NTIC además del texto escrito, como se viene experimentando en la Pontificia Universidad Católica del Perú (PUCP) en sus segundas especialidades y el Ministerio de 
Educación en su plan piloto de educación secundaria en el Programa Huascarán ${ }^{1}$.

Para contribuir a la mejora permanente de la calidad de la oferta universitaria, es necesario diversificarla, atendiendo a diversos sectores de la población y desarrollando una cultura de excelencia en todas las instancias y niveles de la docencia y la investigación universitaria. Para ello será necesario diseñar, entre otras, las siguientes estrategias:

- Desarrollo de competencias en el procesamiento de información en los docentes y estudiantes.

- Incorporación efectiva de las nuevas tecnologías de información y comunicación (NTIC) a la docencia e investigación.

- Promoción de la educación virtual, abierta y a distancia, en sus diferentes combinaciones.

- Fomento a los procesos de innovación pedagógica y curricular.

En la UNMSM se vienen realizando diversos esfuerzos para desarrollar experiencias tendientes a incorporar las NTIC en la docencia universitaria, con resultados satisfactorios pero limitados, que tienen las siguientes características:

- Se hacen pequeños proyectos a nivel de facultades.

- Son proyectos de educación presencial con apoyo virtual (se trabaja con Moodle).

- No tienen el debido control de la calidad educativa.

- Dependen de la voluntad del profesor.

- No hay una remuneración específica por el esfuerzo del profesor.

La necesidad de crear sinergias y mejorar el impacto de las NTIC en el proceso de enseñanza aprendizaje (P/E/A) en la UNMSM ha determinado, recientemente, la creación de una Oficina Central de Educación Virtual de la UNMSM con el propósito de impulsar la virtualización progresiva de la universidad.

\section{1. ¿Por qué emprender la virtualización de la UNMSM?}

Somos conscientes que la enseñanza que se viene impartiendo tradicionalmente en la cuatricentenaria Universidad Nacional Mayor de San Marcos no responde plenamente a las exigencias de la sociedad actual, y consideramos que es urgente emprender la virtualización de la universidad como estrategia para el proceso de innovación y modernización educativa.

Por otra parte, la mayoría de los docentes siguen aplicando estrategias metodológicas tradicionales que desconocen los nuevos paradigmas pedagógicos y didácticos que se apoyan en las NTIC y su aplicación educativa, convirtiéndose éste en uno de los aspectos más críticos de su formación. Lo anterior revela la necesidad impostergable de generar espacios de formación para los docentes en el uso pedagógico, didáctico e integral de las NTIC en el P/E/A en la universidad.

La UNMSM, la decana de América, debe adoptar medidas estratégicas que le permitan responder a las nuevas tendencias de las NTIC para poder competir con sus pares de Iberoamérica, ofreciendo alternativas de acceso al conocimiento que sean diferentes a las formas tradicionales; ése es el caso de la educación virtual.

En nuestro país la educación superior es muy limitada; aunque en todos o en casi todos los departamentos existen universidades públicas y las privadas se masifican cada vez más, a la gran mayoría de población que anualmente egresa de secundaria se le ofrecen relativamente pocas oportunidades de acceder a ella, por lo tanto es sólo un porcentaje menor de jóvenes postulantes los que logran ingresar a las universidades, porcentaje que ha decrecido en el tiempo a nivel nacional, tanto en universidades privadas como públicas. El promedio de ingresantes al sistema universitario nacional es de menos del $20 \%$ del total de jóvenes, con tendencia al decrecimiento, sin considerar la educación de adultos para el trabajo y para toda la vida.

La virtualización constituye una oportunidad para concretar algunos ideales que han estado a la espera de escenarios convenientes. Alguna de esas ideas son las de la Escuela Nueva y el impacto de la Sociedad del Conocimiento. En las siguientes líneas se sintetizan lo que podríamos llamar las debilidades de la educación tradicional, detectadas por el movimiento Escuela Nueva²:

1 Marrou Roldán, Aurora (2001). El post grado a distancia en las universidades de Lima metropolitana: Estudio, diagnóstico y alternativas.

2 Filho, L. (1994). Introducción al estudio de la escuela nueva. Buenos Aires, Kapelusz. 
- Aislamiento del entorno. La escuela tradicional se centra demasiado en sí misma, olvidando su entorno como referencia y como escenario de su impacto.

- Ciencia como sistema cerrado. La escuela tradicional toma la ciencia como un sistema autosuficiente que no entiende ni escucha a otros saberes.

- Libro de texto como fuente exclusiva. Existen fuentes de conocimiento que no son necesariamente documentos o libros de texto y que la escuela tradicional no utiliza.

- Oferta educativa homogénea. Una educación que no tiene en cuenta las diferencias y es por eso inflexible.

- Rol protagónico del maestro. La figura del maestro es tan potente que cierra la entrada a otros actores, incluido el alumno mismo.

- Contenidos informativistas más que contenidos que promuevan la participación.

- Comunicación ineficiente. El proceso educativo es asumido en la escuela tradicional sin tener en cuenta las condiciones de una comunicación eficiente.

A estas debilidades se puede contraponer las virtudes del nuevo escenario tecnológico. Tenemos entre otras:

- Construcción social del conocimiento. La virtualidad, lo hemos visto, facilita la interactividad y la participación, y le da valor a los aportes de los distintos actores del proceso.

- Flexibilidad. Con la adecuada aplicación de las NTIC, la oferta académica se hace más abierta y personalizada y facilita los procesos de retroalimentación / personalización / acompañamiento.

- Educación centrada en el alumno. Al darse una alta interactividad, el maestro pasa a ser un facilitador y así el proceso de aprendizaje se puede centrar en el verdadero sujeto, que es el alumno.

- Visualización de lo aprendido. No sólo en el sentido de una educación más audiovisual, sino en el sentido de una constante conciencia y aplicación inmediata de lo aprendido

- Acercamiento. Conocimiento / comunicación. La aplicación de las NTIC ha permitido sobre todo entender el proceso de aprendizaje como un proceso de comunicación.

Otra de las motivaciones por las que la UNMSM debe emprender el proceso de virtualización es para incrementar su oferta educativa de calidad, para tener presencia a nivel nacional e internacional por ser la universidad más antigua de América, con un prestigio ganado y un liderazgo en todos los campos del conocimiento, para responder así a las nuevas demandas educativas.

\section{2. ¿Cómo virtualizar la Universidad?}

¿Qué entendemos por virtualización universitaria? Según Silvio ${ }^{3}$ se asume como:

“... el fenómeno mediante el cual, gracias a la extensión de la digitalización, tanto los objetos como los procesos y fenómenos propios del quehacer educativo, pueden adquirir una existencia virtual, materializada a través de instrumentos electrónicos, lo cual supone la alteración de las tradicionales relaciones (maestro / alumno, libro / documento, usuario / servicio) que dominaron hasta nuestros días el campo de las funciones institucionales universitarias (docencia, investigación y extensión)..."

Desde el punto de vista técnico, virtualizar la universidad es sobre todo virtualizar sus espacios funcionales, esto es, disponer sectores del ciberespacio para apoyar o sustituir tecnológicamente las actividades académicas y administrativas realizadas físicamente en los espacios tradicionales, de modo que su virtualidad, en los términos arriba descritos, se potencie. Ejemplos de esta nueva configuración son las llamadas aulas, laboratorios, bibliotecas, espacios de encuentro, oficinas, etc., todos ellos virtuales.

Debemos hablar entonces de un campus virtual, cuyo esquema podría vincular y soportar espacios funcionales virtualizados: en el aula virtual, la transferencia de conocimiento; en el laboratorio virtual, la generación de conocimiento; en la biblioteca virtual, la conservación e intercambio de conocimiento, y en la oficina virtual, la gestión general del conocimiento. Pero así como se virtualizan los espacios, la virtualización de la universidad implica sobre todo apoyar tecnológicamente los procesos universitarios hasta potenciar su virtualidad: la enseñanza/aprendizaje,

3 Silvio, José (2000). La virtualización de la universidad. Caracas, UNESCO IESCAL, Colección Respuesta.

4 Silvio, José. Op. cit. 
la investigación, la interacción con el entorno, la gestión y la codificación y recuperación de información. Pero sobre todo, virtualizar la universidad es virtualizar las relaciones que se establecen en una comunidad, en este caso la llamada por muchos, comunidad virtual de aprendizaje ${ }^{4}$. Estas relaciones se distinguen por el alto grado de interactividad entre los miembros de la comunidad, el grado de focalización de sus temas de discusión y por su cohesión social.

La virtualización de la universidad no es sólo un asunto técnico sino, sobre todo, un asunto cultural. Lograr que la comunidad universitaria se apropie de las dinámicas conceptuales y de las bondades de la virtualización, así como de sus manifestaciones tecnológicas, exige una combinación de estrategias; la primera de las cuales consiste en persuadir a los actores del valor que se agrega a su cadena de producción académica tradicional al utilizar las NTIC

Una estrategia muy efectiva consiste en mostrar las bondades de las herramientas virtuales en la construcción de comunidades y en la promoción del trabajo en red, no sólo para los profesores sino para los estudiantes y toda la comunidad universitaria. Desde un punto de vista práctico, la universidad debe estar en capacidad de disponer la infraestructura física y lógica necesaria, así como de los servicios telemáticos, pero sobre todo de los contenidos y la capacitación de sus diferentes actores en sus nuevos roles, así como estrategias de organización pertinentes, estrategias financieras y de costo adecuadas, siendo necesario -tambiénelaborar un Plan Estratégico de Desarrollo de la Educación Virtual en la UNMSM.

\section{ANTECEDENTES}

\section{Universidad Nacional Mayor de San Marcos, la Decana de América}

La Universidad Nacional Mayor de San Marcos, Decana de América, fundada el 12 de mayo de 1551 por Real Cédula firmada por Carlos V, fue el inicio de la historia universitaria del continente. Los dominicos en sus conventos del Cuzco, principal ciudad peruana en el siglo XVI, y de Lima, estudiaban artes y teología para ejercitar a los antiguos miembros y preparar a los novicios de la orden.
La universidad inició funciones el 2 de enero de 1553 en la Sala Capitular del Convento del Rosario de la Orden de los Dominicos, con la concurrencia de la Real Audiencia presidida por el Licenciado Andrés Cianca y por el enviado de la Corona Don Cosme Carrillo, primer miembro laico del cuerpo docente.

La UNMSM ha forjado en sus claustros a un sin número de personalidades notables del arte, la ciencia y la política del Perú y América, quienes desarrollaron sus capacidades al igual que la mayoría de los profesionales y estudiosos que prestan sus servicios en las principales universidades, empresas e instituciones nacionales y extranjeras.

En sus 457 años de vida institucional nuestra universidad ha sido centro generador y difusor de las ciencias, las técnicas, las humanidades, las artes y la cultura nacional e internacional, razón por el cual siempre buscó mantener un nivel de desarrollo acorde con las exigencias en educación e investigación del país y del mundo.

En este contexto del mundo globalizado y la revolución científico-tecnológica que se vive actualmente, es necesario emprender nuevas estrategias de formación y desarrollo utilizando las ventajas competitivas que ofrecen las NTIC, que ha motivado el relanzamiento de la educación a distancia, con la incorporación de nuevas herramientas tecnológicas y avances en las teorías de aprendizaje.

\section{Impacto de las NTIC en el sistema educativo peruano}

Para indagar sobre el grado de desarrollo temático alcanzado en la educación virtual en el Perú, se realizó un levantamiento de información sobre los antecedentes de la inserción de las nuevas tecnologías de información y comunicación (NTIC) en el sistema educativo peruano.

En un análisis de primer nivel se observa una lenta penetración causada por el rezago educativo que afecta al país, debido a que la brecha digital viene asociada a la brechas educativas y de ingresos que afectan al pueblo peruano ${ }^{5}$.

En nuestro país las cabinas públicas resultaron ser un aliado muy importante para las actividades educativas basadas en Internet; sin embargo, la penetración de banda ancha necesaria para esas actividades nos ubica en los últimos lugares en Latinoamérica ${ }^{6}$.

5 Ministerio de Educación. (2002). Educación para la Democracia. Lineamientos de Política Educativa 2001-2006. Lima.

6 Ver www.idc.com. 
En cuanto a las experiencias exitosas en educación a distancia (EaD), e-learning educativo y corporativo, y educación virtual en general, se observa mayor participación de las universidades particulares por la infraestructura tecnológica actualizada de que disponen. Las universidades nacionales de la alianza estratégica vienen realizando investigaciones en educación virtual, internet y modelos pedagógicos para el proceso de modernización, virtualización e innovación educativa.

La mayoría de las universidades en el Perú están usando el sistema e-learning como apoyo a la docencia presencial, aunque existen universidades que ya están adoptando la modalidad semipresencial, o sea el $b$-learning como alternativa de formación.

En el país existen algunos avances en el campo del e-learning y los weblogs corporativos que se vienen desarrollando en el sector empresarial, especialmente para programas de capacitación de personal y de marketing. Con el desarrollo acelerado de las NTIC en los países más avanzados, se viene ampliando aún más la brecha digital que éstos tienen respecto a los países en vías de desarrollo. Es necesario que el Estado intervenga mediante políticas de apoyo con programas gubernamentales de integración de las NTIC al sistema educativo nacional, relanzando programas como Huascarán o Amauta que se encuentran colapsados.

Respecto a la política informática del país, hasta ahora el gobierno actual de Alan García Pérez no ha manifestado su visión respecto a la integración de las NTIC al sistema educativo peruano, no obstante la crisis educativa que afecta al país. Por otra parte, existen vacíos legales en la norma que rige la educación virtual, que es urgente superar.

Para el proceso de modernización e innovación académica de las universidades peruanas es necesario impulsar la innovación, virtualización y flexibilización pedagógica y curricular en los programas de formación profesional.

\section{Programas gubernamentales de inserción de las NTIC en el sistema educativo peruano}

\section{a. El Proyecto Amauta}

En el primer gobierno de Alan García Pérez (1985-1990), se empezó a impulsar una política de informatización de la sociedad peruana mediante el proyecto de los módulos educativos de cómputo (MEC).

Objetivos del proyecto:

- Informatizar a la sociedad peruana para orientar a los agentes de cambio y desarrollo a estructurarse utilizando la informática. Desde luego que se trata de un objetivo a largo plazo y muy ambicioso que no se alcanzó.

- Proporcionar bienestar y trabajo a través de la informática, incentivando la creatividad de las personas e instituciones, para orientar a la sociedad y enfrentar con éxito el desafío del futuro.

Conceptualizando este último objetivo general, es posible decir que se "busca fundamentalmente la informatización de la educación y la educación en informática". Más adelante se dice que la iniciativa del proyecto "Amauta" se toma por decisión presidencial.

La implementación inicial se llevó a cabo en los colegios secundarios estatales de las zonas urbanas de alta concentración poblacional, ampliándolo progresivamente a otros niveles de educación.

En lo referente a la personalización del proceso enseñanza-aprendizaje, el Proyecto Amauta define esto como “... un proceso que pasa a manos del alumno, quien le puede imprimir su propio ritmo" $\mathrm{y}$ al docente se le concibe como un consejero, guía de un nivel más alto de aprendizaje.

En la incorporación de nuevas tecnologías existen dificultades de diversa índole como la experimentación, la creación de nuevos materiales, impacto en la organización del trabajo y formación de docentes. La selección de un sistema coherente para la enseñanza requiere, como tareas previas, que se determinen las necesidades instruccionales del sistema educativo y que se identifiquen las estrategias educacionales antes de adquirir equipos computacionales. Para este proyecto se adquirieron equipos de diversas marcas como Thomson, Apple, IBM, entre otras, sin los requisitos previos señalados. No se realizó la formación de docentes ni el estudio de los marcos teóricos apropiados.

Este proyecto no fue sostenible puesto que la docencia no se apropió del proyecto, participando escasamente; no se hizo un estudio previo de los requerimientos educacionales ni bases pedagógicas. 


\section{b. El Programa Huascarán}

Durante el gobierno de Alejandro Toledo se lanzó el Programa Huascarán como programa estratégico del Estado Peruano para apoyar la consecución de sus fines más altos, expresados en la Carta Magna. Con este programa, la comunidad educativa tendría acceso a la sociedad global de la información, lo que permitiría acortar la brecha digital existente entre la educación urbana y rural, así como ampliar los servicios educativos del nivel secundario a las zonas de frontera.

La misión del Programa Huascarán es "integrar las NTIC al sistema educativo peruano"para promover el desarrollo humano sustentable del pueblo peruano, con especial énfasis en las comunidades rurales de extrema pobreza, reduciendo las brechas educativas e incorporando los principios y valores postulados en el Acuerdo Nacional.

La utilización de las NTIC para enfrentar las inequidades sociales y económicas y así aliviar la pobreza, debe ser contemplada en programas y planes locales, nacionales y regionales y, por ende, en el Programa Huascarán.

El Programa Huascarán logró algunas metas, especialmente en el medio rural, mediante la etapa de sensibilización del Proyecto así como cierta reducción de la brecha digital pero, sin embargo, fue cancelado.

En la actualidad no existen programas gubernamentales del nivel de proyectos nacionales que impulsen la modernización e innovación educativa mediante las NTIC, no obstante el rezago educativo que afecta al Perú.

\section{Penetración de Internet en el Perú}

En 1994, el Estado decidió vender las únicas dos empresas de telecomunicaciones estatales del país: la Compañía Peruana de Teléfonos (CPT) y la Empresa Nacional de Telecomunicaciones (ENTEL) a Telefónica de España, con lo cual no sólo privatizaba el sector sino que constituía un monopolio por cinco años de las líneas telefónicas y las llamadas de larga distancia, nacionales e internacionales. Esto trajo consigo una evidente mejora en la infraestructura y en los servicios de telecomunicaciones. Según recientes estadísticas de OSIPTEL, entre 1993 y 2001 el número de líneas instaladas de telefonía fija se triplicó a 2'022,265; el tiempo de espera para la conexión de una línea se redujo de 18 meses a 15 días; la cuota tope de conexión se redujo de US\$1,500 a US\$ 156; las líneas con fibra óptica crecieron de 200 a 8,173 $\mathrm{km}$ y las localidades con servicio telefónico aumentaron de 1,450 a 3,246 , siendo beneficiadas muchas comunidades del interior del país.

En el caso de Internet se diversificaron los proveedores del servicio y también las ofertas. Un ISP puede ofrecer una conexión a Internet sin costo e incluso proveer una cuenta de correo gratuita, y otro puede ofrecer sus servicios a un precio promedio de US\$ 60 anuales (sólo el acceso al servicio de Internet, porque el consumo telefónico es un pago aparte). Aún así el acceso a Internet es limitado en los hogares ya que sólo 7.75 de cada 100 habitantes tiene una línea telefónica y sólo 4.79 de cada cien habitantes tiene una computadora en casa. Frente a las limitaciones de recursos, tanto económicos como tecnológicos, se ha encontrado una forma de acceder a Internet que es la cabina de internet, un modelo peruano de acceso a Internet internacionalmente conocido. Con un pago promedio de US\$ 0.53 la hora (mucho menos del costo de conectarse desde el hogar), el $89 \%$ de usuarios de Lima accede a Internet por esta vía. Cabe señalar que actualmente se calcula que existen unas 2000 cabinas de Internet que permiten el acceso a la red de redes a aproximadamente 2 millones de peruanos; esto coloca a nuestro país como uno de los que tiene mayor penetración de Internet en la región.

Por otro lado, abierto el mercado de las telecomunicaciones en 1995, el Estado se ha propuesto promover el incremento de la densidad a 20 líneas telefónicas por cada cien habitantes; el acceso a 5000 nuevas ciudades a través de telefonía rural, así como continuar con el mejoramiento de los servicios y la implementación de redes digitales.

No obstante a que las cabinas de Internet en el Perú mejoran los indicadores de penetración de Internet, sin embargo la penetración de banda ancha que es clave para la educación virtual nos ubica en el último lugar en Latinoamérica, como se muestra en la siguiente estadística (ver Cuadro N. $\mathrm{o}$ ):

Cuadro N. 1 . Penetración de banda ancha en Latinoamérica.

\begin{tabular}{|l|l|}
\hline Perú & $1.5 \%$ \\
\hline Chile & $6.0 \%$ \\
\hline Argentina & $2.7 \%$ \\
\hline Brasil & $2.7 \%$ \\
\hline
\end{tabular}

Fuente: IDC, 2006 


\section{Integración de las NTIC en la UNMSM}

A partir del año 2001, la UNMSM se propone contar con una infraestructura tecnológica. Si bien es cierto ésta fue creada el 12 de mayo de 1995, no había sido implementada en toda su potencialidad. Actualmente la UNMSM cuenta con un sistema de comunicaciones que enlaza, mediante más de $16 \mathrm{~km}$. de cable de fibra óptica, a las Facultades y oficinas administrativas entre sí, integrando y compartiendo los recursos de todas las microcomputadoras que están conectadas al sitema; sin embargo el sistema no es del todo eficiente debido a problemas de banda ancha.

\section{a. Red Telemática}

La misión fundamental de la Red Telemática es el diseño, implementación y mantenimiento de los elementos que constituyen lo que podemos llamar la infraestructura informática de la universidad, entendiendo por tal a los elementos físicos, lógicos, configuraciones y procedimientos necesarios para proporcionar a toda la comunidad universitaria los servicios informáticos necesarios para desarrollar sus actividades.

Actualmente la universidad cuenta con una infraestructura tecnológica (hardware) adecuada, por lo que se está trabajando en la implementación de servicios telemáticos en la parte administrativa y docente. Mencionaremos algunos servicios académicos virtuales que brinda la Universidad:

- Cybertesis. Es una iniciativa auspiciada por la UNESCO, la Universidad de Chile y la Universidad de Lyon. Esta iniciativa, dirigida en Perú por la Biblioteca Central de la Universidad Nacional Mayor de San Marcos (UNMSM), busca desarrollar e implementar procesos de digitalización y publicación electrónica de las tesis y otros documentos, utilizando para ello estándares internacionales como OAI-PMH, TEI Lite, Dublin Core, ETD-MS, XML, entre otros. Este proceso, que se inició en junio del 2004 , busca también promover e incentivar a las demás instituciones productoras de tesis a la publicación de estos documentos en texto completo por Internet, contando para ello con la respectiva autorización de los autores. Es así que la Biblioteca Central de la UNMSM viene celebrando convenios y desarrollando talleres de capacitación para la utilización de esta metodología.
- Sistemas de Bibliotecas. La UNMSM cuenta con un sistema de bibliotecas en proceso de integración con todas las Facultades y en formato digital, además de contar con una hemeroteca virtual, revistas de investigación, artículos de investigación y las tesis en formato digital.

Un resumen del diagnóstico de la situación actual del grado de inserción de las NTIC en el sistema educativo de la UNMSM podemos visualizarlo en la siguiente Matriz FODA que se realizó en un Seminario Taller de docentes y estudiantes, llevado a cabo en octubre del 2007 (ver Cuadro № 2). Como se podrá apreciar para maximizar las fortalezas y las oportunidades es urgente la formación de formadores en las NTIC, a fin de que se cree el efecto multiplicador y la réplica en la capacitación de los recursos humanos.

Se espera que la Oficina de Educación Virtual de la UNMSM, de reciente creación, sea el motor de desarrollo de los recursos humanos para el proceso de virtualización de la universidad más antigua de América.

Existen iniciativas muy dispersas en los diferentes sectores y Facultades respecto a la integración de las NTIC al proceso de enseñanza-aprendizaje y a la virtualización de las diferentes tareas en la universidad; sin embargo, no existe un sistema central con objetivos claros y estratégicos a nivel de toda la universidad y que tenga efecto sinérgico. La implementación de la Oficina Central de Educación Virtual puede optimizar el proceso de virtualización de la UNMSM, integrando los diversos subsistemas y racionalizando los recursos.

\section{b. El Proyecto San Marcos Virtual}

Mediante la RR № 01547-R-08 se crea la Oficina Central de Educación Virtual de la UNMSM, como resultado de un dictamen elaborado por una Comisión del Consejo Universitario conformada mediante la RR № 05226-R-03 del 25 de septiembre de 2003.

El Proyecto San Marcos Virtual permitirá desarrollar una cultura emprendedora en la organización así como generar la racionalidad económica administrativa necesaria para la formulación, financiamiento, gestión y evaluación permanente de las alternativas de crecimiento y diversificación de la institución; así como el desarrollo intensivo del potencial humano 
Cuadro № 2. Matriz FODA de diagnóstico de la situación actual de la inserción de las NTIC en el sistema educativo de la UNMSM.

\section{MATRIZ FODA}

\begin{tabular}{|c|c|c|}
\hline $\begin{array}{l}\text { FACTORES } \\
\text { EXTERNOS }\end{array}$ & \begin{tabular}{|l|} 
Lista de Fortalezas \\
F1. La universidad cuenta con \\
docentes con alto grado de \\
especialización en las NTIC. \\
F2. El trabajo realizado por \\
algunos grupos de docentes \\
en impulsar el uso de las \\
NTIC en la UNMSM. \\
F3. Existencia de la política de \\
compra institucional de \\
equipos. \\
F4. Ofrecimiento de cursos de \\
NTIC a la comunidad.
\end{tabular} & $\begin{array}{l}\text { Lista de Debilidades } \\
\text { D1. Escaso uso de las NTIC en procesos de } \\
\text { docencia e investigación } \\
\text { D2. Escasez de personal docente especializado } \\
\text { en Educación Virtual. } \\
\text { D3 Falta de planes estratégicos de integración } \\
\text { de las NTIC en la docencia e investigación. } \\
\text { D4. Demoras en trámites administrativos en } \\
\text { algunas dependencias de la universidad. } \\
\text { D5. Falta de planes de capacitación en } \\
\text { informática y telecomunicaciones para los } \\
\text { docentes y funcionarios de la UNMSM. }\end{array}$ \\
\hline $\begin{array}{l}\text { Lista de Oportunidades } \\
\text { O1. Alianzas estratégicas con } \\
\text { Universidades de elite } \\
\text { O2. Uso de las redes avanzadas que } \\
\text { permiten garantizar la calidad de los } \\
\text { servicios. } \\
\text { O3. Implantación en la universidad de la } \\
\text { Oficina de educación Virtual } \\
\text { O4. Implantar en la UNMM la enseñanza } \\
\text { semipresencial y el apoyo con las } \\
\text { NTIC a la modalidad presencial. } \\
\text { O5. Uso de las nuevas tendencias } \\
\text { basadas en software libre para } \\
\text { reducción de costos. }\end{array}$ & $\begin{array}{l}\text { FO (Maxi, Maxi ) } \\
\text { Estrategia para } \\
\text { maximizar tanto las F } \\
\text { como las O. } \\
\text { Fortalecer los } \\
\text { Programas de Formación } \\
\text { de docentes en NTICs. } \\
\text { (O1, O2, F1, F4) }\end{array}$ & $\begin{array}{l}\text { DO (Mini, Maxi) } \\
\text { Estrategia para minimizar las } D \text { y } \\
\text { maximizar las } 0 . \\
\text { Fortalecer los programas de } \\
\text { vinculación (O1, O2, O3,O4, } \\
\text { D1,D2, D3) }\end{array}$ \\
\hline $\begin{array}{l}\text { Lista de Amenazas } \\
\text { A1. Rápida obsolescencia de los } \\
\text { equipos informáticos } \\
\text { A2. El incremento de la competencia } \\
\text { nacional e internacional } \\
\text { A3. Dificultad de garantizar el } \\
\text { cumplimiento de la propiedad } \\
\text { intelectual en la publicación de } \\
\text { contenidos en web. } \\
\text { A5. Seguridad informática }\end{array}$ & $\begin{array}{l}\text { FA (Maxi,Mini ) } \\
\text { Estrategia para } \\
\text { fortalecer las Fy } \\
\text { minimizar las A. }\end{array}$ & $\begin{array}{l}\text { DA (Mini-Mini) } \\
\text { Estrategia para minimizar tanto las } \\
\text { A como las } D \text {. }\end{array}$ \\
\hline
\end{tabular}

docente en un ámbito de integración de la comunidad sanmarquina, el cual permitirá el desarrollo y estímulo para la internacionalización y la presencia nacional de la UNMSM.

La UNMSM cuenta con importantes fortalezas para alcanzar los objetivos del proyecto, principalmente por la capacidad y compromiso de sus docentes, estudiantes y egresados con su institución, en las diferentes áreas del conocimiento y con el reconocido prestigio interna- cional de nuestra casa de estudios en el ámbito nacional e internacional. San Marcos Virtual deberá proponer mecanismos para potenciar de una manera continua las capacidades y habilidades de la comunidad sanmarquina en el uso de las NTIC aplicadas a la educación y a la investigación ${ }^{7}$. El impulso de la Universidad Virtual permitirá consolidar la presencia institucional de la UNMSM, con 457 años de experiencia a nivel nacional e internacional, rescatando su liderazgo en Latinoamérica.

7 Vicerrectorado Académico UNMSM. (2003). Proyecto de Universidad Virtual de San Marcos (UVSM). 
Esta Oficina Central de Educación Virtual (SMVIRTUAL) tiene la siguiente misión, visión y objetivos:

- Misión. Apoyar la educación presencial, la investigación, la extensión y la gestión universitaria para ofrecer a nivel nacional e internacional educación formal y no formal de alta calidad, basada en modelos educativos y tecnológicos innovadores de educación a distancia en modalidad virtual, ajustados a los estándares internacionales.

- Visión. La Oficina Central SMVIRTUAL, en el año 2012, será reconocida a nivel nacional e internacional por la calidad, innovación y pertinencia de su modalidad educativa virtual, por el impacto social de la investigación realizada y la construcción de redes sociales de aprendizaje.

- Objetivo general de la SMVIRTUAL. Consolidar la Oficina Central de Educación Virtual de la UNMSM como líder en educación abierta y a distancia, que actúa con calidad en el ámbito local, nacional e internacional.

- Objetivos específicos de la SMVIRTUAL. Diseñar e implementar el sistema de gestión estratégica para lograr calidad y eficiencia en los diversos procesos de la SMVIRTUAL.

Asegurar la excelencia y calidad de la educación virtual en los diferentes proyectos que realice la SMVIRTUAL.

Asegurar la calidad del sistema de gestión tecnológica que garantice los servicios y la producción de SMVIRTUAL.

Virtualizar progresivamente las actividades fundamentales de la UNMSM: docencia, investigación, administración y extensión universitaria.

\section{JUSTIFICACIÓN DEL TRABAJO DE INVESTIGACION}

La educación virtual ha surgido (en general en las instituciones de educación superior) como una respuesta a la demanda del medio externo, inserto en una sociedad marcada fuertemente por el impacto de las tecnologías de la información y de las comunicaciones en todo el quehacer de las personas, tanto en el ámbito personal como el profesional.
Las instituciones de educación superior han debido adoptar medidas estratégicas que les permitan responder a las nuevas tendencias de la información y comunicación para poder competir con sus pares, ofreciendo alternativas de acceso al conocimiento, diferentes de las formas tradicionales; tal es caso de la educación virtual a distancia o e-learning.

El mundo laboral, por otro lado, ha dejado de ser rutinario, dando paso a una múltiple funcionalidad de tareas, lo que conlleva a un desplazamiento de funciones según las necesidades e innovaciones introducidas en el proceso de producción o venta. Esto repercute en que los trabajadores vean incrementadas y diversificadas las tareas que deben realizar, lo cual implica aprender continuamente; en otras palabras, cobra importancia la formación continua y la necesidad de aprender a lo largo de toda la vida. Por razones de tiempo, la educación virtual a distancia se visualiza como una excelente alternativa para apoyar este tipo de formación.

La UNMSM, a través de la Oficina Central de Educación Virtual, debe responder a los requerimientos impuestos por los nuevos paradigmas en materia de educación, y debe incorporar dentro de las áreas temáticas del Plan General Estratégico de la UNMSM.

El proyecto San Marcos Virtual se encuentra inmerso dentro de los lineamientos principales del Plan Estratégico de la UNMSM llamado Innovación, virtualización y flexibilización pedagógica y curricular para mantener su liderazgo en una dimensión global; lograr la internacionalización y el liderazgo Latinoamericano y, al mismo tiempo, agregar valor a nuestros servicios educativos con el uso adecuado de las NTIC, una herramienta estratégica para el docente sanmarquino.

Para el proceso de implementación de la Oficina Central de Educación Virtual es de fundamental importancia diseñar un modelo pedagógico, tecnológico y organizacional adecuado a la realidad de la UNMSM. Sin embargo esta oficina enfrenta algunos problemas, ya que se creó por recomendación de una Comisión nombrada por el Consejo Universitario, sin un estudio de factibilidad económica, operacional y pedagógica. El presente trabajo pretende rellenar esos vacíos, estructurando los modelos pedagógicos, tecnológicos y organizacionales que demuestren que es viable. 


\section{OBJETIVOS DEL ESTUDIO}

\section{Objetivo General}

El objetivo general de este estudio es el de diseñar un modelo de virtualización para innovar los procesos de formación presencial y de EaD, así como las actividades de investigación y extensión universitaria en la UNMSM.

\section{Objetivos Específicos}

1. Evaluar la factibilidad tanto desde el punto de vista técnico como económico y pedagógico, para desarrollar el e-learning en la UNMSM, como alternativa de educación complementaria y a distancia virtual

2. Diseñar el modelo pedagógico apropiado para la educación virtual en la UNMSM.

3. Diseñar un modelo organizacional para el desarrollo de las actividades de San Marcos Virtual.

4. Realizar un análisis y selección de tecnologías elearning para la administración del aprendizaje.

5. Sensibilizar a la comunidad universitaria de San Marcos sobre este tema.

6. Realizar un diseño para un plan de implementación de un programa de e-learning.

\section{MARCO CONCEPTUAL}

\section{E-learning y blended learning.}

Las nuevas NTIC han permitido a las universidades ofrecer nuevos modelos de enseñanza, que van desde la educación presencial a la educación a distancia (e-learning), permitiendo además desarrollar los denominados modelos integrados o mixtos (b-learning) donde, según plantea Bartolomé ${ }^{8}$, el proceso docente se integra armónicamente con actividades en el aula presencial y en la virtual.

El concepto de virtualización es definido por Quéau ${ }^{9}$ como:

“... un proceso y resultado al mismo tiempo del tratamiento de la comunicación mediante la computadora, de datos, informaciones $y$ conocimientos. Más específicamente, la virtualización consiste en representar electróni- camente y en forma numérica digital, objetos y procesos que encontramos en el mundo real. En el contexto de la educación superior, la virtualización puede comprender la representación de procesos y objetos asociados a actividades de enseñanza y aprendizaje, de investigación y gestión, así como a objetos cuya manipulación permite al usuario, realizar diversas operaciones a través de Internet, tales como aprender mediante la interacción con cursos electrónicos, inscribirse en un curso, consultar documentos en una biblioteca electrónica, y comunicarse con estudiantes y profesores".

Se fomenta la virtualización como innovación estratégica para mejorar la docencia y la investigación. Las nuevas tecnologías y los campus virtuales se presentan como una herramienta fundamental para potenciar el aprendizaje y la colaboración en el proceso científico entre profesores de distintas disciplinas, diferentes universidades y distancias geográficas, pero también en la investigación que pueden realizar conjuntamente profesor y alumno.

\section{Metodología} métodos:

En la investigación se aplicaron los siguientes

- Histórico lógico. Para estudiar la esencia del problema relacionado con la introducción de diferentes entornos virtuales de enseñanza aprendizaje (EVEA) en nuestro medio.

- Encuestas. Para conocer la opinión de la comunidad educativa sobre el uso experimental del prototipo de campus virtual.

- Entrevistas. Para conocer la opinión de los funcionario y docentes sobre el proyecto de virtualización de la universidad.

- Análisis de Documentos. Plan de Desarrollo Estratégico de la UNMSM y otros.

\section{Fundamentos de la enseñanza virtual}

Entre las múltiples aplicaciones de Internet encontramos la enseñanza virtual (también llamada teleformación, enseñanza a través de Internet, enseñanza on-line o e-learning), puesto que las posibilidades de la red son óptimas para esta tarea. Hoy en día, el modelo educativo virtual

8 Bartolomé Pina, Antonio (2004). “Blended Learning. Conceptos básicos”. En: Revista Píxel-Bit № 23, pp. 7-20. http://www.sav.us.es/pixelbit.

9 Queau, Phillipe. (1993). Le virtual. París, Editions Champ Vallon Setina. 
está plenamente implantado en muchas organizaciones, tanto desde su perspectiva tecnológica como didáctica.

La enseñanza virtual requiere de un cambio en el modelo educativo clásico para adaptarse al nuevo entorno de aprendizaje no presencial y basado en las NTIC. Es un error muy común pensar que lo fundamental de esta área es la tecnología.

Los aspectos más directamente afectados por el uso de los medios digitales son la presentación de la información del profesor a los alumnos, el material de estudio y consulta, la interacción profesor-alumno, y el propio proceso de aprendizaje y autoevaluación del alumno.

La globalización de la educación superior viene impulsando cambios paradigmáticos de orientación y métodos de apropiación de conocimiento por parte de los estudiantes; esto implica cambiar de un sistema de enseñanza de aprendizaje centrado en el profesor a un sistema de aprendizaje centrado en el alumno ${ }^{10}$, tal como se ilustra en los siguientes gráficos.

La actual velocidad de creación de nuevos conocimientos rápidamente deja obsoletos los conocimientos impartidos en la mayoría de las carreras profesionales, y el docente deja de ser la única fuente de enseñanza del alumno; por lo tanto, los nuevos roles del profesor se expresan en organizar un entorno de aprendizaje que le permita al alumno disponer de las condiciones y motivaciones para aprender a estudiar, aprender a asimilar conocimientos y aprender a desarrollar sus competencias.

Es más importante que el alumno se apropie de un método y una visión de la evolución del conocimiento que le permita seguir aprendiendo a lo largo de toda la vida activa, con el fin de continuar siendo un profesional vigente y actualizado

\section{SOBRE EL DISEÑO DE UN MODELO PEDAGÓGICO}

1. Propuesta pedagógica de un modelo para los proceso de formación a distancia o presencial mediante plataformas tecnológicas.

En una primera aproximación una propuesta pedagógica puede consistir en una estrategia global para el proceso de incorporación de las NTIC en el P/E/A en la universidad.

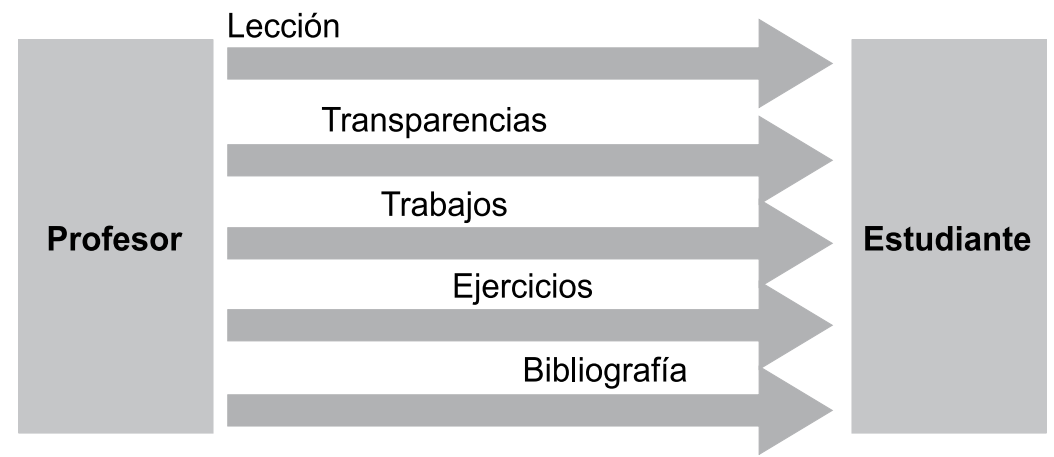

Gráfico N. $^{\circ}$ 1. Modelo educativo tradicional centrado en el docente

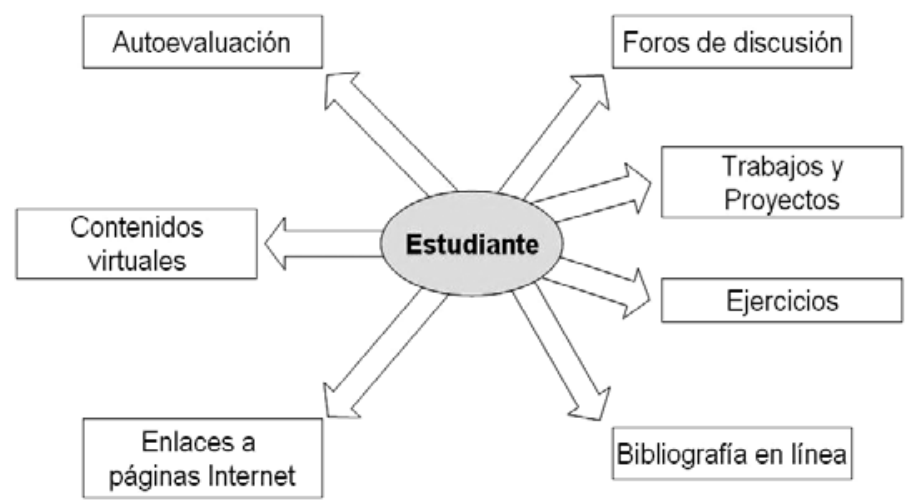

Gráfico $\mathrm{N}^{\circ}$ 2. Modelo educativo centrado en el estudiante 
La propuesta busca la integración de las NTIC al entorno de la enseñanza-aprendizaje, coordinando las diferentes líneas de acción en torno a un modelo de integración, mediante el uso de las tecnologías y recursos asociados que resulten apropiados.

Para contar con un modelo pedagógico fundamentado en el constructivismo, que asegure el cumplimiento de los objetivos de la educación virtual en la UNMSM, será necesario diseñar y llevar a la práctica un proceso de enseñanza-aprendizaje caracterizado por el uso de medios informáticos y telemáticos como recurso didáctico y elemento mediador para el aprendizaje, vale decir, un modelo teórico que oriente con claridad sobre las formas de organizar un ambiente de aprendizaje para la docencia universitaria. El modelo comprende el conjunto de elementos pedagógicos, metodológicos, evaluativos y estructurales, sobre los cuales se fundamentan y fundamentarán las asignaturas o programas que se pretendan virtualizar.

El abordaje del proceso enseñanza-aprendizaje se realiza bajo una mirada holística del estudiante, en el cual se atiende con igual interés a la adquisición de competencias cognitivas, actitudinales, de habilidades y éticas así como a las características de los contextos internacionales, nacionales y locales en los que se desenvuelve y desenvolverá el estudiante. Esta interrelación entre el contexto y el proceso educativo es parte fundamental del éxito de un modelo educativo del siglo XXI, ${ }^{11}$ pues desarrolla no solo las competencias que se requieren en el aula, sino aquellas que se requieren para desenvolverse con éxito en toda la vida.

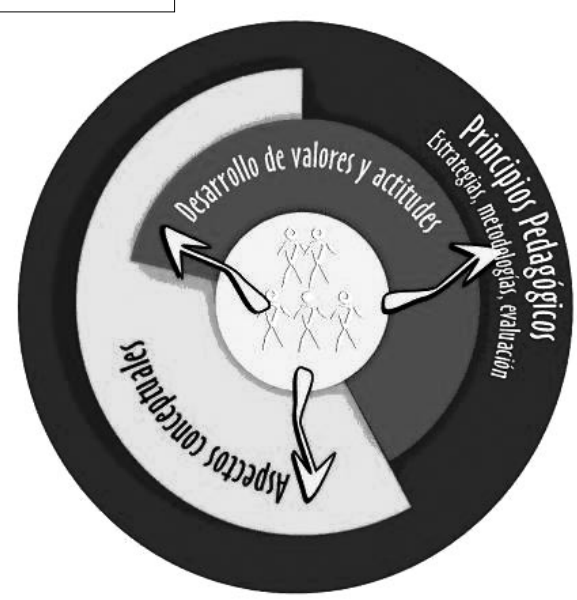

Gráfico № 3. Modelo Pedagógico para los proceso de formación a distancia o presencial mediante plataformas tecnológicas.
Respondiendo a esa intencionalidad, el modelo educativo asume el desarrollo de tres dimensiones (principios pedagógicos, desarrollo de valores y actitudes, y aspectos conceptuales), como se observa en el siguiente gráfico (ver Gráfico N.우 3).

Diseñar un modelo pedagógico consiste en elegir una serie de principios que permitan sustentar la forma en que se llevará a cabo el proceso de enseñanza-aprendizaje. En este proceso interactúan tres elementos: unos contenidos, unos alumnos y un profesor (ver Gráfico №4). Debemos observar en el esquema algo fundamental: las líneas que representan las relaciones entre profesores-contenido-estudiante son todas de doble dirección ya que los tres elementos juegan un papel esencial en todos los procesos, en contra de las prácticas tradicionales. Un modelo pedagógico debe dar luces para decidir sobre los tres elementos y su comportamiento; debe ofrecer información sustentada que permita responder a las siguientes preguntas fundamentales:

- ¿Qué se debe enseñar?

- ¿Cuándo enseñar?

- ¿Cómo enseñar?

- ¿Qué, cuándo y cómo evaluar?12

\section{SOBRE EL DISEÑO DEL MODELO ORGANIZACIONAL.}

Se puede resumir esta organización como la estructuración de las relaciones que deben existir en las funciones, niveles y actividades de los elementos humanos y materiales para que un organismo social, como lo es la Oficina de Educación Virtual de la UNMSM (SMVIRTUAL), pueda lograr la máxima eficiencia en la realización de sus planes y objetivos.

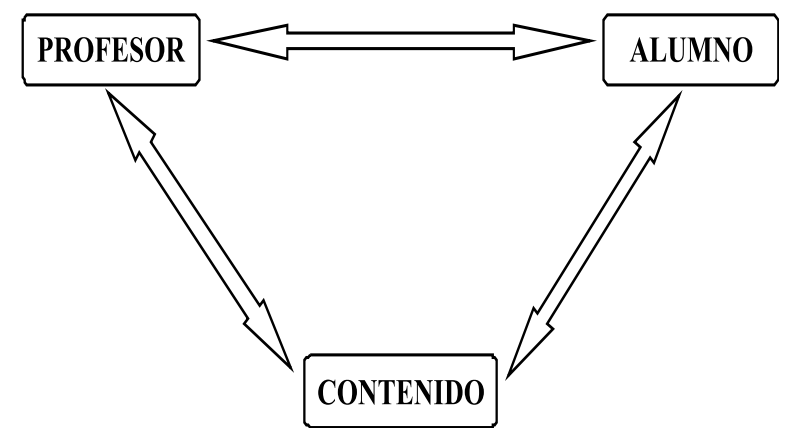

Gráfico № 4. Elementos de un modelo pedagógico

11 Hasblady Segovia, C. (2002). Aciertos y oportunidades de crecimiento en el proceso de implantación de ambientes virtuales de aprendizaje en la Universidad de La Sabana-Colombia.

12 Gonzáles, M.A. (2000). Modelos Pedagógicos para un ambiente de aprendizaje con NTIC. Medellín, Conexiones. 
La Oficina de Educación Virtual de reciente creación, dependerá del Rectorado, tendrá un Director Ejecutivo con funciones delegadas por el Rector, un Administrador del Campus Virtual y tres directores en las áreas pedagógicas de Tecnologías, Administración y Servicios.

Su estructura se establece mediante niveles (ver Gráfico N. 5):

- Nivel 0. Encargado de la dirección ejecutiva del proyecto.

- Nivel 1. Encargado de los espacios funcionales, en los que se distinguen las funciones pedagógicas y didácticas, las tecnológicas y las de Administración y Servicios.

- Nivel 2 o de grupos de trabajo. Conformado por grupos de trabajo de especialistas en diferentes disciplinas, entre las que se destacan el diseño instruccional, las telecomunicaciones, el desarrollo de software y la gestión.

- Nivel 3 o de subgrupos encargados de actividades específicas o comisiones de estudio y/o trabajo.

\section{SOBRE LAS PLATAFORMAS TECNOLÓGICAS}

Existen en el mercado un gran número de plataformas de teleformación (del orden de varios cientos de miles) que presentan distintas funcionalidades y enfoques pedagógicos. Hay varias de ellas muy conocidas y extendidas como WebCT, BlackBoard, FirstClass o Lotus Learning Space. También existen soluciones de software libre de muy buena calidad como ILIAS, Moodle, Clarolin, etc. En la universidad se viene usando la plata- forma libre Moodle por ser la más ergonómica. A mediano plazo la universidad puede diseñar su plataforma propia.

1. Análisis comparativo de algunas plataformas interactivas de código abierto más conocidas en nuestro medio.

A partir de la implementación de la Plataforma Tecnológica se investigó el ámbito de las nuevas tecnologías incorporadas al quehacer educativo, y se adoptó la plataforma constructivista Moodle. La decisión se fundamentó en el estudio comparativo de distintas plataformas de e-learning y el análisis teórico de la bibliografía seleccionada ${ }^{13}$.

\section{Características técnicas de las herramientas}

Una de las características de Internet y del campo de los computadores en general, es el continuo cambio. La flexibilidad y capacidad de adaptarse al cambio debe ser una característica de las herramientas que pueden venir definidas por:

- Compatibilidad entre plataformas.

- Tipo de plataforma que utiliza el servidor.

- Utilización de estándares.

- Posibilidades multimedia.

- Interfaz de usuario ergononómico.

- Posibilidad de consultar los materiales off-line.

- Almacenamiento de los contenidos en una base de datos.

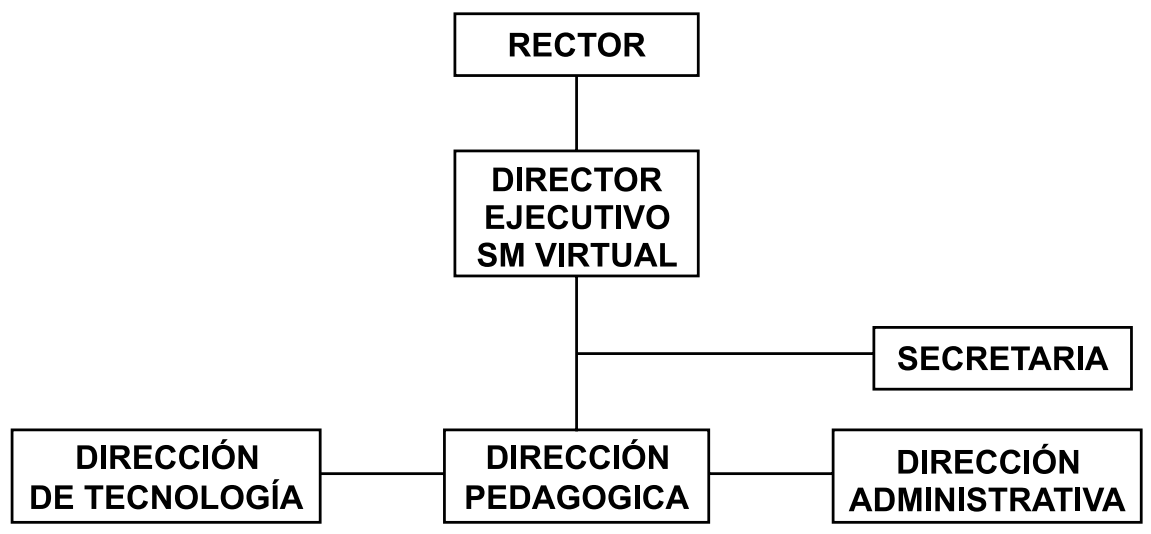

Gráfico N. ${ }^{\circ}$ 5. Organización de la Oficina de Educación Virtual de la UNMSM (SMVIRTUAL)

13 Bedriñana Ascarza, Aquiles. (2006). Las NTIC en la innovación educativa de la Facultad de Ciencias Administrativas de la UNMSM: experiencias de virtualización de la docencia. Bilbao. 
- Acceso a materiales o contenidos de otros cursos dentro del mismo servidor.

3. Descripción de las principales plataformas de códigos libres

Estas son las plataformas de libre distribución que actualmente existen en Internet con suficiente documentación:

- Claroline. A lo largo del mundo hay muchas instituciones que utilizan esta plataforma. Paquete software que permite a los profesores crear, administrar y añadir sus cursos a través de la web. Utiliza sistemas operativos como Linux, Unix, Windows, Mac OS X.

- Fle3. Existen usuarios por todo el mundo, normalmente instituciones universitarias. Es un entorno de aprendizaje basado en la web y diseñado para soportar estudiantes y grupos cuyo trabajo se centre en la creación y desarrollo de expresiones del conocimiento. Utiliza los sistemas operativos de Linux, Mac OS X, y Windows.

- Ilias. Son muchas las instituciones que usan esta plataforma en todo el mundo, ya que permite a los usuarios crear, editar y publicar cursos. Funciona bajo los sistemas operativos Linux y Sun Solaris.

- Moodle. Desarrollada por Martin Dougiamas como parte de su tesis doctoral, y cuenta con más de 10,855 sitios en 152 países, en 60 idiomas. Estas cifras convierten a Moodle en una de las plataformas de e-learning más utilizadas a nivel mundial, con una enorme cantidad de usuarios $(102,493)$ intercambiando experiencias y desarrollando y perfeccionando este sistema. Es un paquete de software diseñado para ayudar a los proyecto de sistemas informáticos especializados en la educación a distancia y semipresencial. Opera bajo diversos sistemas operativos como Unix, Linux, Windows, Mac OS $X$ y Netware.

- ATutor. Es un entorno de creación y gestión de cursos en línea. Pone mucho énfasis en la accesibilidad de los materiales de aprendizaje, y funciona bajo los sistemas operativos de Unix, Linux, Windows, Mac OS X y Netware

Se realizó un análisis comparativo de las plataformas de código libre (ver Cuadro N. ${ }^{\circ} 3$ ), teniendo en cuenta sus elementos y características técnicas y educativas ya analizadas para el modelo de universalización, teniendo en cuenta, fundamentalmente, los costos. Se utilizó la siguiente escala:

- 2 puntos cuando el elemento está completamente implementado.

- 1 punto cuando el elemento no está del todo implementado.

- 0 puntos cuando no está implementado.

Indiscutiblemente las plataformas que ofrecen más ventajas son Atutor y Moodle, pero la plataforma Moodle, al menos en las versiones utilizadas para la comparación, es superior a las otras.

\section{BENEFICIOS ESPERADOS DEL PROCESO DE VIRTUALIZACIÓN DE LA UNMSM}

\section{Soporte virtual de actividades académicas}

- Ofrecer un servicio adicional a los estudiantes universitarios, quienes tendrán acceso a los materiales de clase y estarán comunicados con docentes y compañeros a toda hora y desde cualquier lugar.

- Involucrar a los docentes universitarios a las nuevas tecnologías de la información y comunicación (NTIC).

- Facilitar la implementación de los planes pedagógicos y el seguimiento de las actividades académicas.

- Facultar la participación más proactiva de los estudiantes en su propia educación, mediante herramientas que fomenten la indagación y la participación, incluso fuera de horarios curriculares.

- Simplificar las tareas del docente al evitar la constante preparación de copias impresas para ser entregadas en clase, y de los estudiantes, quienes pueden enviar sus trabajos a través de Internet sin necesidad de acercarse hasta la institución con una copia impresa de los mismos.

- Mejorar la comunicación entre docentes y alumnos y garantizar que la información que comparten los alumnos sea uniforme.

- Generar una retroalimentación permanente acerca del grado de evolución de los alumnos en cada materia, permitiendo un seguimiento más personal previo a la instancia evaluatoria del examen parcial o final. 
Cuadro $\mathrm{N}^{\circ}$ 3. Análisis comparativo de las plataformas de código libre

\begin{tabular}{|l|c|c|c|c|c|}
\hline \multicolumn{1}{|c|}{ Aspecto comparado } & Claroline & Fle3 & Ilias & Moodle & ATutor \\
\hline Idiomas (indispensable el español) & 1 & 1 & 1 & 2 & 1 \\
\hline Accesibilidad & 0 & 0 & 0 & 2 & 1 \\
\hline Recursos multimedia & 1 & 1 & 2 & 1 & 1 \\
\hline Control de acceso & 2 & 2 & 2 & 2 & 2 \\
\hline Correo electrónico & 2 & 0 & 2 & 2 & 2 \\
\hline Tablón de anuncios & 1 & 0 & 2 & 2 & 2 \\
\hline Sencillez de la interfaz & 1 & 1 & 1 & 2 & 2 \\
\hline Conocimientos previos de informática & 0 & 2 & 2 & 2 & 2 \\
\hline Importación de material de cualquier tipo & & & & & \\
incluido ejecutables & 1 & 0 & 1 & 2 & 2 \\
\hline Seguimiento del estudiante & 1 & 1 & 2 & 2 & 2 \\
\hline Copias de seguridad & 0 & 0 & 0 & 2 & 0 \\
\hline Elementos que permiten la interactividad & 2 & 2 & 2 & 2 & 2 \\
\hline Colaboración entre profesores & 0 & 0 & 0 & 2 & 0 \\
\hline Documentación & 1 & 1 & 1 & 2 & 1 \\
\hline TOTAL & $\mathbf{1 3}$ & $\mathbf{1 1}$ & $\mathbf{1 8}$ & $\mathbf{2 7}$ & $\mathbf{2 0}$ \\
\hline
\end{tabular}

\section{Como soporte virtual de educación a distancia}

- Permitir el seguimiento de los estudiantes en la fase no presencial de los programas de educación a distancia que desarrolle la universidad.

- Facilitar el acceso a los cursos y capacitación de alumnos, evitándose los traslados, las demoras y las pérdidas de tiempo.

- Permitir que exista interacción entre el alumno y el docente.

- Generar una administración descentralizada de los espacios disponibles para cada docente, sin depender excesivamente del equipo de sistemas ni consumir recursos internos de la institución.

- Incrementar la capacidad de respuesta del docente a los problemas puntuales de sus alumnos.

- Facilitar la implementación de los planes curriculares.

- Facilitar la función de guía o tutor de los docentes de cada uno de los cursos.

- Facilitar el rol más activo por parte del estudiante, con una mejor administración de los tiempos.

- Facilitar el rol del docente, quien podrá realizar una mejor utilización de sus capacidades, quien no "dicta" conocimientos sino que orienta y despeja dudas.

- Facilitar un método práctico y económico de distribución de contenidos académicos respecto a las alternativas tradicionales.

- Permitir que los docentes posean una idea cabal de la evolución y el nivel de participación de los alumnos a lo largo del programa de las carreras y cursos, lo que posibilita la concentración de los esfuerzos en aquellas áreas en las que existen dificultades.

- Evitar el desplazamiento físico de las personas motivando para que compartan una fuente común de conocimientos, la posibilidad de participar en discusiones abiertas con sus pares, así como lograr que estén continuando informados acerca de la realidad de las clases que están tomando.

- Posibilitar que los alumnos tomen los cursos a su propio ritmo y en el momento que consideren más conveniente, lo que facilita trabajar y estudiar al mismo tiempo.

- Potenciar las posibilidades de los alumnos para participar y expresar opiniones y puntos de vista, tornándose en protagonistas centrales de su propia formación y de la de sus compañeros.

- Facilitar la actualización de los contenidos. 
3. Como soporte de la educación continua y capacitación de los docentes

- Estandarizar las necesidades de capacitación de docentes y facilitar la implementación de un programa progresivo que se iniciaría brindando soporte a las actividades cotidianas del docente.

- Acercar las nuevas tecnologías a la experiencia diaria del docente, facilitando la incorporación de nuevas actividades y nuevas tecnologías simultáneamente, que lo ayuden en su función docente.

- Capacitar docentes en el proceso de creación de espacios virtuales, de manera incremental y permanente.

- Difundir las experiencias de los docentes e incentivar el efecto multiplicador.

- Desarrollar un foro con la demostración y ejemplificación de casos de éxito de uso de la plataforma. Consejos útiles y fuerte énfasis en las ventajas del sistema para el docente.

- Desarrollar e incorporar un manual de uso como parte de la plataforma.

- Instalación y operación de un curso modelo que oriente a los docentes sobre las potencialidades del sistema.

- Brindar soporte al docente en los aspectos pedagógicos y de tecnología informática en apoyo a sus cursos.

- Emitir reportes periódicos acerca del uso que cada docente hace de las herramientas disponibles.

- Desarrollar reuniones periódicas de grupos de docentes que comparten sugerencias y analizan aprendizajes.

4. Como plataforma de apoyo a la investigación

- Facilitar el acceso a las redes virtuales de información académica y de investigación.

- Facilitar el acceso y participación en grupos temáticos virtuales.

- Facilitar información relativa a la elaboración de proyectos de investigación.

- Establecer enlaces con fuentes de financiamiento.

- Facilitar una plataforma virtual para el desarrollo de proyectos académicos.
HACIA EL DISEÑO DE UN PLAN DE DESARROLLO

DE LA EDUCACIÓN VIRTUAL DE LA UNIVERSIDAD NACIONAL MAYOR DE SAN MARCOS

La elaboración de un Plan Estratégico para el Desarrollo de la Educación Virtual y a Distancia en la UNMSM permitirá, a partir de sus actuales fortalezas y debilidades, orientar las acciones institucionales para establecer una oferta de programas académicos para la formación inicial y continua de profesores y estudiantes.

Los objetivos del desarrollo de este Plan Estratégico de la Educación Virtual y a Distancia en la UNMSM pueden ser:

- Identificar las fortalezas y debilidades institucionales en el ámbito de la educación a distancia en los nuevos escenarios para el aprendizaje con TIC, en relación a las oportunidades y amenazas del medio externo local y las ofertas de programas académicos extranjeros.

- Plantear un conjunto de políticas y metas para el desarrollo institucional organizado de la educación virtual y a distancia para un horizonte de cinco años.

La virtualidad es la expresión de dos grandes aspiraciones y estrategias del sector educativo: ampliación de cobertura y mejoramiento de la calidad.

Como ha sucedido en otros campos, la aplicación de las tecnologías digitales a la educación se ha desarrollado desde dos vertientes estratégicas: una primera vertiente estratégica, la más conocida y extendida, consiste en aplicar las nuevas tecnologías a cursos y programas de formación y capacitación para estudiantes remotos o a distancia. La otra vertiente se refiere específicamente al desarrollo de programas académicos a distancia soportados con tecnología virtual, a los cuales denominaremos simplemente educación a distancia/virtual, pues su evolución tiene como punto de partida la que hoy puede denominarse tecnología tradicional de educación a distancia (módulos en papel, apoyados en casetes de audio y video e interacción vía postal, telefónica o con tutores).

\section{CONCLUSIONES}

1. Para el proceso de modernización e innovación académica de la Universidad Nacional Mayor de San Marcos (UNMSM), decana de América, es necesario consolidar los procesos 
de innovación, virtualización y flexibilización pedagógica y curricular en los programas de formación, en correspondencia con las nuevas formas de apropiación, producción y difusión del conocimiento para el desarrollo humano sustentable, teniendo como motor de desarrollo a la Oficina de Educación Virtual de la UNMSM.

2. En cuanto a la factibilidad pedagógica, el creciente número de personas que ingresan a los programas de pre y post grado en línea, es una muestra de que la educación virtual es una respuesta válida y oportuna a la actual demanda de alternativas de acceso al conocimiento, diferentes a las formas tradicionales de educación-aprendizaje. La educación virtual ha demostrado ser apropiada para la formación continua y el aprendizaje a lo largo de toda la vida, debido a las ventajas competitivas que ofrece en cuanto al ahorro de tiempo, costos y desplazamientos. La educación virtual no es excluyente con la educación presencial, sino que es un complemento de la misma. La mayoría de los programas de formación de pre y post grado están siendo soportados por plataformas tecnológicas, aulas virtuales y laboratorios virtuales.

3. En cuanto a la factibilidad técnica, actualmente la mayoría de las universidades peruanas ya vienen utilizando las NTIC y principalmente Internet, para apoyo de sus procesos tradicionales.

4. Las plataformas de código libre, como Moodle, vienen demostrando eficiencia. Desde el punto de vista económico es recomendable usar inicialmente un software libre y, posteriormente, desarrollar un software propio.

\section{RECOMENDACIONES}

1. La virtualización de la UNMSM debe ser progresiva, empezando por el pre y postgrado y el soporte a la docencia presencial y la biblioteca.

2. La formación de docentes en educación virtual es prioritaria.

3. Formular el Plan de Desarrollo Estratégico de la Integración de las NTIC a la docencia y la virtualización progresiva de la UNMSM.
4. Aprovechar las experiencias exitosas de la red de macrouniversidades que integra la UNMSM.

5. Promover la investigación en las NTIC para la docencia universitaria.

6. Formación de recursos humanos en educación virtual.

\section{BIBLIOGRAFÍA}

Bartolomé Pina, Antonio (2004). "Blended Learning. Conceptos básicos". En: Revista Píxel-Bit №23, pp. 7-20. http://www.sav. us.es/pixelbit.

Bedriñana Ascarza, Aquiles. (2006). Las NTIC en la innovación educativa de la Facultad de Ciencias Administrativas de la UNMSM: experiencias de virtualización de la docencia. Bilbao.

Estrategia Integral de Virtualización de la docencia de la Universidad de Los Lagos-Puerto Montt. Agosto 2005.

Filho, L. (1994). Introducción al estudio de la escuela nueva. Buenos Aires, Kapelusz.

Gonzáles, M.A. (2000). Modelos Pedagógicos para un ambiente de aprendizaje con NTIC. Medellín, Conexiones.

Hasblady Segovia, C. (2002). Aciertos y Oportunidades de Crecimiento en el proceso de implantación de ambientes virtuales de aprendizaje en la Universidad de La Sabana-Colombia.

Marrou Roldán, Aurora (2001). El Post Grado a Distancia en las Universidades de Lima Metropolitana: Estudio, Diagnóstico y Alternativas.

Ministerio de Educación. (2002). Educación para la Democracia. Lineamientos de Política Educativa 2001-2006. Lima.

Queau, Phillipe. (1993). Le virtual. París, Editions Champ Vallon Setina.

Silvio, José. (2000). La virtualización de la universidad. Caracas, UNESCO IESCAL, Colección Respuesta. 